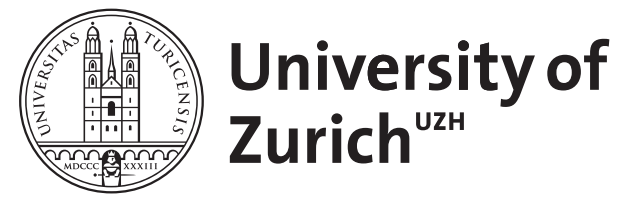

Zurich Open Repository and Archive

University of Zurich

University Library

Strickhofstrasse 39

CH-8057 Zurich

www.zora.uzh.ch

Year: 2015

\title{
Hausärzte vermeiden unnötige Spitaleintritte
}

Djalali, S

Posted at the Zurich Open Repository and Archive, University of Zurich

ZORA URL: https://doi.org/10.5167/uzh-116941

Journal Article

Published Version

Originally published at:

Djalali, S (2015). Hausärzte vermeiden unnötige Spitaleintritte. PrimaryCare:403-404. 


\title{
Hausärzte vermeiden unnötige Spitaleintritte
}

\author{
Sima Djalali, Claudia Berlin, Maud Maessen
}

\begin{abstract}
In einem guten Gesundheitssystem sollten unnötige Spitaleinweisungen selten sein. Eine Analyse in der Schweiz zeigt, dass die Rate der vermeidbaren Spitaleinweisungen mit der regionalen Dichte von Hausarztpraxen zusammenhängt.
\end{abstract}

Als «vermeidbare» Hospitalisationen werden Spitaleinweisungen angesehen, die durch ein Grundleiden verursacht werden, das entweder

- durch Prävention hätte verhindert werden können (z.B. Masern),

- durch frühzeitige Diagnose und Behandlung hätte ambulant behandelt werden können (z.B. Ulkusperforation) oder

- durch kontinuierliches ambulantes Follow-up und Versorgungsmanagement hätte kontrolliert werden können (z.B. Diabetes).

Die Häufigkeit solcher Hospitalisationen wird in der Versorgungsforschung als Surrogat für die Zugänglichkeit und Funktionsfähigkeit der ambulanten Gesundheitsversorgung angesehen. So zeigen Länder wie die Vereinigten Staaten vermeidbare Hospitalisationen vor allem als Ausdruck dessen, dass grosse Teile der Bevölkerung aufgrund des gesellschaftlichen Gefälles, unzureichender Krankenversicherung und regional verschieden verteilter Versorgungsressourcen nur eingeschränkten Zugang zu ambulanter Versorgung haben. Diese Teile der Bevölkerung haben bei akuten Problemen resp. Exazerbationen erst in einem Stadium Kontakt mit dem Gesundheitssystem, in dem eine Hospitalisation unumgänglich ist.

In Regionen mit einer hohen Spezialistendichte unter den niedergelassenen Ärzten war die Rate der vermeidbaren Hospitalisationen höher

\section{Paradies Schweiz?}

Verglichen damit herrschen in der Schweiz paradiesische Zustände: Dank des hohen Einkommensstandards, des Krankenkassenobligatoriums und der hohen medizinischen Ressourcendichte existieren hierzulande praktisch keine Barrieren, die Kranke da- von abhalten, zeitnah ambulante medizinische Hilfe in Anspruch zu nehmen. Dennoch gibt es auch hier regelmässig Fälle von Hospitalisationen, die potentiell vermeidbar gewesen wären. Es stellt sich die Frage, ob und, wenn ja, wieso die ambulanten Versorgungsstrukturen ihrer Funktion, vermeidbare Hospitalisationen zu vermeiden, scheinbar nicht ausreichend nachkommen.

\section{Viele Regionen, viele Varianten}

In einem Gemeinschaftsprojekt des Instituts für Hausarztmedizin der Universität Zürich und des Instituts für Sozial- und Präventivmedizin der Universität Bern wurden das regionale Auftreten potentiell vermeidbarer Hospitalisationen in der Schweiz in den Jahren 2008-2010 genauer untersucht und Faktoren identifiziert, die mit dem erhöhten Auftreten solcher Fälle assoziiert waren. Die Analysen wurden auf Basis von Daten aus der medizinischen Statistik der Krankenhäuser, der Krankenhausstatistik sowie der Volkszählung des Bundesamts für Statistik durchgeführt. Um regionale Unterschiede herauszuarbeiten, wurden 59 Einzugsgebiete von Akutspitälern in der Schweiz ermittelt. Wie sich zeigte, variierte die Inzidenz potentiell vermeidbarer Hospitalisationen zwischen diesen 59 Regionen stark: Sie betrugen zwischen 274 und 982 vermeidbaren Hospitalisationen pro 100000 Einwohner.

\section{Herzinsuffizienz und COPD führend}

In allen Regionen waren Herzinsuffizienz (durchschnittlich 236 Fälle/100000 Einwohner) und COPD (durchschnittlich 122 Fälle/100 000 Einwohner) die am häufigsten zugrundeliegenden Erkrankungen, die zu potentiell vermeidbaren Hospitalisationen führten. Es folgten Diabetes, Hypertonie und Asthma. Die Häufigkeit aller vermeidbaren Spitalaufenthalte nahm über die Jahre 2008-2010 um 2,7\% zu. 


\section{Starke Präsenz der Hausarztmedizin schützt}

Betrachtet man die regionalen Unterschiede unter Berücksichtigung von demografischen Unterschieden der Bevölkerungen genauer, so zeigt sich, dass potentiell vermeidbare Hospitalisationen in Regionen mit einer hohen Hausarztdichte seltener vorkamen. In Regionen mit einer hohen Spezialistendichte unter den niedergelassenen Ärzten war die Rate der vermeidbaren Hospitalisationen dagegen höher. Weiterhin zeigte sich, dass die Anzahl von vermeidbaren Hospitalisationen in den Regionen höher war, in denen mehr als $42 \%$ der Einwohner in einer ländlichen Region lebten, verglichen mit Ballungsgebieten ohne ländlichen Bevölkerungsanteil. Die regionale Dichte von Spitalbetten hatte in dieser Studie keinen Einfluss auf die Rate der vermeidbaren Hospitalisationen.

\section{Eine Frage der Prioritäten}

Die Ergebnisse der Studie könnten ein Hinweis darauf sein, dass in Gesundheitssystemen mit unbeschränktem Zugang die individuelle Entscheidungsschwelle der ambulant tätigen Ärzte dafür ausschlaggebend ist, ob ambulant beherrschbare Gesundheitsprobleme ambulant behandelt werden oder zu einer Spitaleinweisung führen. Diese Schwelle scheint bei Hausärzten höher angesetzt zu sein als bei Spezialisten, so dass eine hohe Dichte an Hausärzten die Rate vermeidbarer Hospitalisationen senkt. Ein Grund dafür könnte sein, dass Hausärzte es als Kernaufgabe sehen, (Primär-)Prävention zu leisten, gefährliche Verläufe abzuwenden und die langfristige kontinuierliche Betreuung bei chronischen Erkrankungen zu erbringen und damit Hospitalisationen vorzubeugen, während die ambulante Versorgung durch Spezialisten andere Prioritäten setzt und eher punktuell ausgerichtet ist.

Korrespondenz:

Dr. med. Sima Djalali Institut für

Hausarztmedizin Universität Zürich Pestalozzistrasse 24 8091 Zürich sima.djalali[at]usz.ch

\section{Eine Frage der Daten}

Doch diese Hypothese bedarf weiterer Forschung. In der hier vorgestellten Studie war es aufgrund fehlender Daten leider nicht möglich festzustellen, wie er- schöpfend die Grundleiden der Patienten im Vorfeld einer potentiell vermeidbaren Hospitalisation tatsächlich ambulant diagnostiziert und behandelt wurden. Für die Klassifizierung von potentiell vermeidbaren Hospitalisationen war in dieser Studie einzig der Diagnosecode eines Grundleidens ausschlaggebend, bei dem Hospitalisationen nach Definition der Organisation für wirtschaftliche Zusammenarbeit und Entwicklung (OECD) vermeidbar wären. Es werden zusätzliche Daten benötigt, um die Schwere der zur Hospitalisation führenden Symptome sowie die Angemessenheit der vorangegangenen ambulanten $\mathrm{Be}$ handlung einschätzen zu können. Diese Daten müssen dann in Zusammenhang mit den Charakteristika der ambulanten Versorgungsstrukturen in Verbindung gebracht werden, um mit Sicherheit sagen zu können, welche regionalen Unterschiede tatsächlich für die grossen Häufigkeitsunterschiede von potentiell vermeidbaren Hospitalisationen verantwortlich sind.

\section{Literatur}

Berlin C, Busato A, Rosemann T, Djalali S, Maessen M. Avoidable hospitalizations in Switzerland: a small area analysis on regional variation, density of physicians, hospital supply and rurality. BMC Health Serv Res. 2014;14:289.

\section{PrimaryResearch - das Fenster \\ zur Forschung}

In einer Artikelserie stellen wir die Forschungsarbeiten vor, die das Institut für Hausarztmedizin der Universität Zürich (IH$A M Z$ ) seit seiner Gründung publiziert hat. Die Originalarbeiten sind entweder open access zugänglich oder beim jeweiligen Autor, der die Studien hier vorstellen wird, auf Anfrage erhältlich.

Die Ergebnisse geben einen spannenden Einblick in die täglichen Herausforderungen, aber auch die Leistung der Hausarztmedizin.

An dieser Stelle ein herzlicher Dank an alle Kolleginnen und Kollegen, die sich an den jeweiligen Projekten beteiligen und die hier präsentierten Ergebnisse erst ermöglicht haben!
Universität Zürich $^{\text {UzH }}$ Institut für Hausarztmedizin 